\title{
Endochin-like quinolone-300 and ELQ-316 inhibit Babesia bovis, B. bigemina, B. caballi and Theileria equi
}

\author{
Marta G. Silva ${ }^{1 * \dagger}$, Reginaldo G. Bastos ${ }^{1 \dagger}$, J. Stone Doggett ${ }^{2}$, Michael K. Riscoe ${ }^{2}$, Sovitj Pou ${ }^{3}$, Rolf Winter ${ }^{3}$,
} Rozalia A. Dodean ${ }^{3}$, Aaron Nilsen ${ }^{2,3}$ and Carlos E. Suarez ${ }^{1,4^{*}}$

\begin{abstract}
Background: The most common apicomplexan parasites causing bovine babesiosis are Babesia bovis and $B$. bigemina, while B. caballi and Theileria equi are responsible for equine piroplasmosis. Treatment and control of these diseases are usually achieved using potentially toxic chemotherapeutics, such as imidocarb diproprionate, but drugresistant parasites are emerging, and alternative effective and safer drugs are needed. The endochin-like quinolones (ELQ)-300 and ELQ-316 have been proven to be safe and efficacious against related apicomplexans, such as Plasmodium spp., with ELQ-316 also being effective against Babesia microti, without showing toxicity in mammals.

Methods: The inhibitory effects of ELQ-300 and ELQ-316 were assessed on the growth of cultured B. bovis, B. bigemina, B. caballi and T. equi. The percentage of parasitized erythrocytes was measured by flow cytometry, and the effect of the ELQ compounds on the viability of horse and bovine peripheral blood mononuclear cells (PBMC) was assessed by monitoring cell metabolic activity using a colorimetric assay.

Results: We calculated the half maximal inhibitory concentration $\left(\mathrm{IC}_{50}\right)$ at $72 \mathrm{~h}$, which ranged from 0.04 to $0.37 \mathrm{nM}$ for ELQ-300, and from 0.002 to $0.1 \mathrm{nM}$ for ELQ-316 among all cultured parasites tested at $72 \mathrm{~h}$. None of the parasites tested were able to replicate in cultures in the presence of ELQ-300 and ELQ-316 at the maximal inhibitory concentration $\left(\mathrm{IC}_{100}\right)$, which ranged from 1.3 to $5.7 \mathrm{nM}$ for ELQ-300 and from 1.0 to $6.0 \mathrm{nM}$ for ELQ-316 at $72 \mathrm{~h}$. Neither ELQ-300 nor ELQ-316 altered the viability of equine and bovine PBMC at their $\mathrm{IC}_{100}$ in in vitro testing.

Conclusions: The compounds ELQ-300 and ELQ-316 showed significant inhibitory activity on the main parasites responsible for bovine babesiosis and equine piroplasmosis at doses that are tolerable to host cells. These ELQ drugs may be viable candidates for developing alternative protocols for the treatment of bovine babesiosis and equine piroplasmosis.
\end{abstract}

Keywords: Bovine babesiosis, Equine piroplamsosis, Babesia bovis, Babesia bigemina, Babesia caballi, Theileria equi, Endochin-like quinolones, ELQ-300, nnELQ-316

\footnotetext{
*Correspondence: marta_silva@wsu.edu; carlos.suarez@usda.gov

${ }^{\dagger}$ Marta G. Silva and Reginaldo G. Bastos contributed equally to this work

${ }^{1}$ Department of Veterinary Microbiology and Pathology, College of Veterinary Medicine, Washington State University, Pullman, WA, USA

Full list of author information is available at the end of the article
}

\section{Background}

Tick-borne diseases caused by apicomplexan hemoparasites, such as those of genera Babesia and Theileria, impose serious economic impact on the cattle and horse industries worldwide [1, 2]. Babesiosis and theileriosis share similar acute disease signs, including anemia, loss of weight, anorexia and fever [3]. As a general rule, Babesia and Theileria are not eliminated in surviving animals and

(c) The Author(s) 2020. This article is licensed under a Creative Commons Attribution 4.0 International License, which permits use, sharing, adaptation, distribution and reproduction in any medium or format, as long as you give appropriate credit to the original author(s) and the source, provide a link to the Creative Commons licence, and indicate if changes were made. The images or other third party material in this article are included in the article's Creative Commons licence, unless indicated otherwise in a credit line to the material. If material is not included in the article's Creative Commons licence and your intended use is not permitted by statutory regulation or exceeds the permitted use, you will need to obtain permission directly from the copyright holder. To view a copy of this licence, visit http://creativeco mmons.org/licenses/by/4.0/. The Creative Commons Public Domain Dedication waiver (http://creativecommons.org/publicdomain/ zero/1.0/) applies to the data made available in this article, unless otherwise stated in a credit line to the data. 
therefore can cause lifelong persistent infections. Important shared features among Babesia and Theileria species include a sexual reproductive cycle in their Ixodes arthropod hosts and asexual reproduction in the red blood cells (RBC) of their vertebrate hosts, a process that results in severe, potentially fatal hemolytic anemia [4-6].

Theileria equi and Babesia caballi are the etiological agents of equine piroplasmosis (EP), a disease that affects horses, mules, donkeys and zebras worldwide [7]. The threat of EP imposes severe and costly restrictions in the transportation of high-performance horses between endemic and non-endemic areas for participation in equestrian sporting events $[3,6]$. No vaccines are currently available against $T$. equi and B. caballi, and considerable resources have been spent to develop drugs to treat animals against the harmful effects of acute EP and to prevent the loss of performance in chronically infected, high-value horses. Despite these efforts, horses that survive acute infection, especially when caused by T. equi, become persistently infected, asymptomatic carriers, a condition that can be associated with the resurgence of outbreaks of EP worldwide [8].

Babesia bovis and B. bigemina are two main causative agents of bovine babesiosis (BB), an acute and persistent economically important disease of cattle that typically causes high mortality [1]. While B. bigemina is usually associated with relatively milder acute hemolytic disease, $B$. bovis is implicated in a more severe presentation of the acute phase of the disease, characterized by the cytoadhesion of parasite-infected RBC in the brain capillaries, similar to that seen in cerebral malaria, and often leads to death [1].

Prevention and control of EP and BB have been typically achieved by controlling tick vector populations, the use of live attenuated vaccines in the case of $\mathrm{BB}$ and chemotherapy. The live, attenuated vaccines available to prevent acute $\mathrm{BB}$, which are in use only in a limited number of countries, are only recommended for animals aged $<1$ year and present several additional constraints, including the risk of reversion to virulence. Furthermore, cattle vaccinated with live, attenuated vaccines may also become persistently infected with the parasites and can serve as a reservoir for tick acquisition and transmission [9]. In addition, live vaccines can cause severe disease to immunocompromised and older cattle which may be more susceptible to the attenuated vaccine strains [9]. Given these scenarios, some animals vaccinated with live Babesia vaccines also need to be treated with anti-babesial drugs to prevent the development of acute disease caused by virulent escapes within the population of parasites in the attenuated vaccine strains. Currently, babesicidal drugs are the only option available for preventing losses due to babesiosis in adult vaccine-susceptible animals that need to be transported from non-endemic Babesia areas to endemic ones. Altogether, these aspects highlight the importance of having reliable babesicidal drugs to control the spread of outbreaks and prevent development of acute disease in herds vaccinated with live, attenuated Babesia vaccines.

Chemotherapy treatments based on diminazene aceturate and imidocarb dipropionate are the most effective and first-choice methods to manage animals with acute $\mathrm{BB}$ and EP $[10,11]$. However, the efficacy of these drugs is highly variable, and treated animals need to be monitored closely for adverse effects, especially when high doses are used in attempts to achieve clearance of the parasites, which is a usual occurrence for valuable horses affected by EP [12]. In addition to toxic side effects, and although specific resistance to imidocarb by Babesia and Theileria parasites has not yet been documented, the potential for the development of drug resistance by Babesia parasites to other drugs, such as amicarbalide isethionate, has been previously recorded [13]. Consequently, there is the need to search for new effective and less toxic alternative chemotherapeutics against BB and EP.

Endochin-like quinolone (ELQ) compounds are potent selective inhibitors of the mitochondrial cytochrome $b c_{1}$ complex, as demonstrated in Plasmodium falciparum, the causative agent of the most severe form of human malaria [14-17]. ELQ compounds have been shown to be highly effective against different species and multiple stages of Plasmodium [18, 19]. Importantly, the ELQ300 and ELQ-316 compounds have been selected as preclinical anti-malaria candidates based on their reasonable oral bioavailability at efficacious doses, long half-life and metabolic stability $[18,19]$. A recent study also demonstrated the efficacy of ELQ prodrugs combined with atovaquone to treat experimental babesiosis caused by Babesia microti in the immunodeficient mouse model [20]. Data from the study reported here showed that the combined therapy of ELQ and atovaquone resulted in complete clearance of the parasite, with no disease recrudescence even $>100$ days after discontinuation of the treatment [20]. Based on these conclusive parasite inhibitory results, we evaluated the effect of ELQ-300 and ELQ-316 on the in vitro growth of B. bovis, B. bigemina, B. caballi and T. equi. Strong inhibition of the development of all these parasites, coupled with the lack of toxic effects on host cells, suggests that these two compounds are promising candidates for future development of novel alternative therapies to control $\mathrm{BB}$ and $\mathrm{EP}$.

\section{Methods}

\section{Synthesis of ELQ-300 and ELQ-316}

The ELQ-300 and ELQ-316 compounds were synthesized as previously described $[19,21]$ (Additional file 1: 
Figure S1). Both compounds were kindly provided by the Department of Molecular Microbiology and Immunology, Oregon Health and Science University (Portland, OR, USA). Purity of both ELQ derivatives was assessed to be $>99 \%$ by proton-nuclear magnetic resonance spectroscopy and gas chromatography-mass spectrometry. ELQ-300 and ELQ-316 were diluted in 100\% dimethyl sulfoxide (DMSO) to prepare stock solutions. Stock solutions were kept at room temperature until use. Working solutions were freshly prepared in parasite culture medium before being added to the parasite cultures.

\section{Cultures of B. bovis, B. bigemina, B. caballi and T. equi}

Babesia caballi Puerto Rico strain [18], B. bovis Texas strain [19], B. bigemina Puerto Rico strain [20] and T. equi Florida strain [21] were grown in long-term microaerophilous stationary-phase cultures and incubated at $37{ }^{\circ} \mathrm{C}$ in an atmosphere of $5 \% \mathrm{CO}_{2}, 5 \% \mathrm{O}_{2}$ and $90 \% \mathrm{~N}_{2}$, as previously described [22-25]. Babesia bovis and B. bigemina were grown in 96-well plates, in $180 \mu \mathrm{l}$ per well of complete HL-1 culture media (pH 7.2; 2.38 g/l HEPES, $5 \mathrm{ml} / \mathrm{l} \mathrm{L}$-glutamine, $60 \mathrm{U} / \mathrm{ml}$ of penicillin G, $60 \mu \mathrm{g} / \mathrm{ml}$ of streptomycin and $0.15 \mu \mathrm{g} / \mathrm{ml}$ of amphotericin B; SigmaAldrich, St. Louis, MO, USA) supplemented with $40 \%$ bovine serum. Cultures contained a suspension of 10 and $5 \%$ packed cell volume of bovine erythrocytes for B. bovis and B. bigemina, respectively. Babesia caballi and T. equi were cultured under similar conditions, but the culture media were supplemented with 10 and $20 \%$ horse serum, respectively. In addition, $B$. caballi and T. equi cultures contained a suspension of $10 \%$ packed cell volume equine erythrocytes.

\section{Parasite growth inhibition assay}

Growth inhibition assays using ELQ-300 or ELQ-316 were performed on cultured $B$. bovis, B. bigemina, $B$. caballi and $T$. equi with a starting percentage of parasitized erythrocytes (PPE) of 0.2. Parasites were grown as described above in culture media containing different concentrations of ELQ-300 or ELQ-316 (range 0.05 to $50 \mathrm{nM}$ ) diluted in DMSO. Parasite cultures in the presence of DMSO $(0.5 \mu \mathrm{l})$ and in the absence of the ELQ compounds were used as a positive control for parasite growth. Extra wells containing uninfected bovine or equine RBC were prepared and used as negative controls for the flow cytometric analysis. Fresh culture medium (150 $\mu \mathrm{l} /$ well) containing the respective drug concentration was replaced daily to parasite cultures. These experiments were carried out in triplicate for each tested concentration and controls, over a period of $72 \mathrm{~h}$. PPE was monitored daily by flow cytometry, as previously described [22, 23]. The half maximal (50\%) inhibitory concentration $\left(\mathrm{IC}_{50}\right)$ values were calculated for ELQ-300 and ELQ-316 at 24, 48, and $72 \mathrm{~h}$ of incubation by extrapolation, with a $50 \%$ reduction of the PPE in the wells containing the ELQs compared with the positive control wells using nonlinear regression (GraphPad Prism ver. 8.0.2 for Windows; Graphpad Software Inc., San Diego, CA, USA). Similarly, maximal (100\%) inhibitory concentration $\left(\mathrm{IC}_{100}\right)$ values were also calculated at $72 \mathrm{~h}$.

\section{Flow cytometric analysis for detection of parasite growth in cultures}

The PPE of parasite cultures was determined by flow cytometry, as previously described $[26,27]$. Briefly, $5 \mu \mathrm{l}$ of the cultures was collected from the bottom of the wells and centrifuged at $450 \mathrm{~g}$ for $1 \mathrm{~min}$ at $4{ }^{\circ} \mathrm{C}$. The supernatant was discarded and the cell pelle washed twice with $150 \mu \mathrm{l}$ of phosphate buffer saline (PBS) $\mathrm{pH}$ 7.2, following which the cell pellet was suspended in $200 \mu \mathrm{l}$ of $25 \mu \mathrm{g} /$ $\mu \mathrm{l}$ hydroethidine (HE) (Invitrogen, Carlsbad, CA, USA), incubated in $5 \% \mathrm{CO}_{2}$ in an incubator at $37{ }^{\circ} \mathrm{C}$ for $20 \mathrm{~min}$ in the dark and ten washed twice with $200 \mu \mathrm{l}$ of PBS to remove the excess HE. The supernatant was then discarded, and the cell pellet was suspended in $200 \mu$ of fresh PBS. The suspended cells were analyzed by flow cytometry using a Guava ${ }^{\circledR}$ easyCyte flow cytometer (Luminex Corp., Austin, TX, USA) at a ratio of 800-1000 cells/ $\mu \mathrm{l}$ with 20,000 events collected. The results were analyzed by FCS Express v6 (De Novo Software, Glendale, CA, USA). Normal, uninfected horse and cattle $\mathrm{RBC}$ were used as a negative control for the flow cytometric analysis.

\section{Effect of ELQ-300 and ELQ-316 IC $\mathrm{I}_{100}$ on parasite growth}

In vitro growth inhibition assays were performed over a period of 8 days using a starting PPE of 0.2 and $2 \%$. Parasites were grown in the presence of calculated ELQ-300 or ELQ-316 $\mathrm{IC}_{100}$ values. Cultures growing in medium in the absence of the ELQs and non-infected RBC maintained in medium only were used as positive and negative controls, respectively. Culture medium containing the respective compound concentrations was replaced daily, $150 \mu \mathrm{l}$ medium per well, for a period of $72 \mathrm{~h}$, following which the parasites were cultivated in media only and split every $48 \mathrm{~h}$ for a period of 8 days. The PPE was evaluated at 24,48 , and $72 \mathrm{~h}$, and 8 days of culture by flow cytometry.

\section{Cytotoxicity assay}

Cytotoxicity of ELQ-300 and ELQ-316 in ex vivo peripheral blood mononuclear cells (PBMC) of bovine and equine were examined by exposing cells to the compounds at their calculated $\mathrm{IC}_{50}$ and $\mathrm{IC}_{100}$. For the bovine PBMC experiment, ELQ-300 $\mathrm{IC}_{50}$ and $\mathrm{IC}_{100}$ were $0.56 \mathrm{nM}$ and $4.3 \mathrm{nM}$, respectively. And for the ELQ-316 $\mathrm{IC}_{50}$ and $\mathrm{IC}_{100}$ were 0.07 
$\mathrm{nM}$ and $3.92 \mathrm{nM}$, respectively. For the equine PBMC experiment, ELQ-300 $\mathrm{IC}_{50}$ and $\mathrm{IC}_{100}$ were 0.23 and $5.94 \mathrm{nM}$, respectively, and ELQ-316 $\mathrm{IC}_{50}$ and $\mathrm{IC}_{100}$ were 0.11 and $6.18 \mathrm{nM}$, respectively. Viability of bovine and horse PBMC was evaluated by monitoring cell metabolic activity using a colorimetric assay. Briefly, peripheral blood was collected from healthy cattle and horses via jugular venipuncture into Vacutainer ${ }^{\circledR}$ tubes containing ACD (acid citrate dextrose) (Becton, Dickinson and Company, Franklin Lakes, NJ, USA) and PBMC were isolated using Histopaque ${ }^{\circledR}$ (SigmaAldrich) per standard protocol. Cells were then plated at $2 \times 10^{4}$ cells/well in 96-well plates in complete Dulbecco's modified essential medium (cDMEM; 10\% fetal bovine serum, 24 mM of HEPES, $2 \mathrm{mM}$ of L-glutamine, $100 \mathrm{IU} /$ $\mathrm{ml}$ penicillin, and $100 \mathrm{ug} / \mathrm{ml}$ streptomycin) and incubated with the ELQ compounds. The Cell Proliferation WST-1 reagent (Roche Applied Science, Penzberg, Germany) was added to the cell cultures following the manufacturer's protocol at 24, 48, and $72 \mathrm{~h}$ after exposure to the ELQ compounds. Absorbance at $440 \mathrm{~nm}$ was measured using an enzyme-linked immunosorbent assay (ELISA) plate reader at $4 \mathrm{~h}$ after adding the WST-1 reasgent to the cells. Cells in cDMEM in the absence of the ELQ compounds and cells exposed to DMSO only (1/400 dilution, which corresponds to the highest volume used on the diluted ELQs) were used as negative controls. PBMC exposed to concanavalin (Con) A diluted in cDMEM ( $5 \mu \mathrm{g} / \mathrm{ml})$ (Sigma-Aldrich) and $\operatorname{Draxxin}^{\circledR}[22]$ were used as a positive control.

\section{Statistical analysis}

Growth of parasites in culture was analyzed using oneway analysis of variance (GraphPad Prism version 8.0.2 for Windows; Graphpad Software Inc.). Values of $P<0.05$ were considered to be statistically significant in terms of the effect of the ELQs on the parasite growth. Significant differences in PBMC viability were measured by Student's t-test, and $P$ values $<0.05$ were considered to be significant.

\section{Ethical Statement}

The in vitro cultures of B. caballi, T. equi, B. bovis and $B$. bigemina require erythrocyte and serum equine and cattle donors. The protocols used for the bleeding of the horse and cattle donors for were approved by the Institutional Animal Care and Use Committee (IACUC) of the University of Idaho (Project title: Bovine and Equine Bleeding, UI IACUC \# 2020-42).

\section{Results and discussion}

ELQ-300 and ELQ-316 inhibit the growth of B. bovis, $B$. bigemina, B. caballi and T. equi

The effect of ELQ-300 and ELQ-316 on parasite growth, with a starting PPE of $0.2 \%$, was evaluated using seven different concentrations of each compound, ranging from 0.05 to $50 \mathrm{nM}$. Both tested drugs significantly inhibited $(P<0.05)$ the growth of $B$. bovis, B. bigemina, B. caballi and T. equi (Figs. 1a-d, 2a-d). In addition, the inhibitory effect of ELQ-300 and ELQ-316 was found to be dose-dependent for all four parasites tested. The calculated $\mathrm{IC}_{50}$ and $\mathrm{IC}_{100}$ values of ELQ-300 and ELQ-316 for each parasite are shown in Table 1. Overall, comparisons of the $\mathrm{IC}_{50}$ values among all parasites tested indicate increased susceptibility to ELQ-316 compared to ELQ300. The ELQ-316 $\mathrm{IC}_{50}$ varied from 0.002 to $0.1 \mathrm{nM}$, while the ELQ-300 $\mathrm{IC}_{50}$ varied from 0.04 to $0.37 \mathrm{nM}$, as measured at $72 \mathrm{~h}$ of culture (Table 1 ).

Interestingly, our calculated values of $\mathrm{IC}_{50}$ for ELQ-300 and ELQ-316 are in the same range or lower than values estimated for other related apicomplexans in previous studies. ELQ-316 $\mathrm{IC}_{50}$ values of 7.97, 0.66 and $0.35 \mathrm{nM}$ were established for Besnoitia besnoiti and Toxoplasma gondii tachyzoites, respectively [28, 29]. In addition, a previous study demonstrated ELQ-300 $\mathrm{IC}_{50}$ values of 15.4 and 23.1 $\mathrm{nM}$ for Plasmodium knowlsei and P. falciparum, respectively [15]. In addition to the acceptable $\mathrm{IC}_{50}$ inhibitory values found for ELQ-300, our study showed even lower ELQ-316 $\mathrm{IC}_{50}$ values for B. bovis, B. bigemina, B. caballi and T. equi, suggesting that these parasites are also highly susceptible to these two drugs. The $\mathrm{IC}_{50}$ values obtained for ELQ-300 and ELQ-316 are also lower than those shown with anti-babesial drugs in recently published studies, but in the same $\mathrm{IC}_{50}$ range of imidocarb dipropionate for $B$. bovis and B. bigemina (Additional file 2: Table S1).

ELQ-300 and ELQ-316 consistently completely abrogated the growth of all four parasites when tested at their respective $\mathrm{IC}_{100}$. The calculated $\mathrm{IC}_{100}$ values ranged from 1.3 to $5.7 \mathrm{nM}$ for ELQ-300, and from 1.0 to $6.0 \mathrm{nM}$ for ELQ-316 (Table 1). Overall, B. bigemina, displayed the lowest $\mathrm{IC}_{100}$ value of the four parasites tested and thus appears to be the most susceptible parasite to ELQ300. On the other hand and based on the $\mathrm{IC}_{100}$ values (Table 1), T. equi appears to be more susceptible to ELQ316 than the other four parasites tested in this study. Taking the $\mathrm{IC}_{50}$ and $\mathrm{IC}_{100}$ data together, we conclude that ELQ-300 and ELQ-316 are able to efficiently inhibit the in vitro growth of $B$. bovis, B. bigemina, B. caballi and $T$. equi blood stages. Notably, while the calculated $\mathrm{IC}_{100}$ of T. equi is unexpectedly high (500-fold higher than the $\mathrm{IC}_{50}$ ) (Table 1), we cannot rule out the possibility that the actual concentration of the drug in the culture well was affected by poor solubility in the culture media.

\section{Growth inhibitory effect of ELQ-300 and ELQ-316 is independent of initial parasitemia}

We then tested whether the efficiency of the compounds is dependent on the parasite initial parasitemia, by 


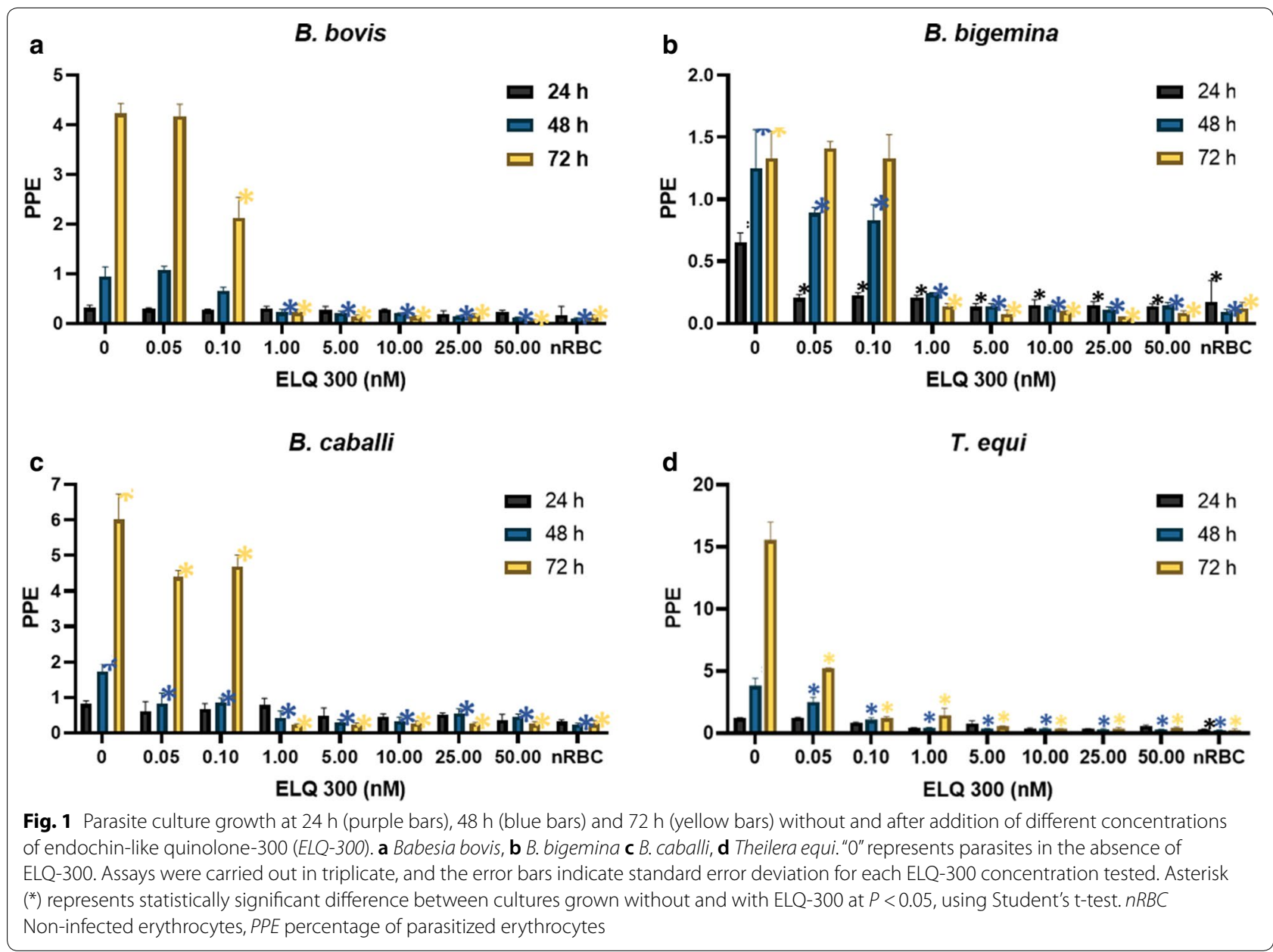

comparing the effects of ELQ-300 and ELQ-316, at their respective $\mathrm{IC}_{100}$, on the four parasites growing in in vitro cultures with starting PPEs of 0.2 and $2 \%$. Neither $B$. bovis, $B$. caballi nor T. equi were able to grow in in vitro cultures in the presence of the ELQ-300 $\mathrm{IC}_{100}$, regardless of their initial PPE $(P<0.05)$ (Fig. 3a, c, d). Nonetheless, the addition of ELQ-300 to B. bigemina cultures at an initial PPE of $2 \%$ did not result in a rapid decrease of parasitemia (Fig. 3b), in contrast to what was found when the initial PPE was $0.2 \%$ (Fig. 3b).

Based on these results, a parasite rescue experiment was performed in which the parasites were grown in culture in the presence of ELQ-300 for 3 days, following which the cultures were split 1:10 and maintained in media free of the drug for an additional 5 days of culture. Parasite growth was not detected $(P<0.05)$ by the end of this period of time for $B$. bovis and T. equi, but that was not the case for B. bigemina and B. caballi (Fig. 3b, c). These results suggest the absence of pre-existing ELQ300-resistant parasite subpopulations in the B. bovis and $T$. equi strains with the ability to survive the initial drug-inhibitory treatment among the parasite strains tested. Collectively, these results are consistent with the relatively increased tolerance of $B$. bigemina and $B$. caballi to ELQ-300, compared to the other two parasites tested, as shown in Fig. 1b and c.

Interestingly, none of the four species of parasites tested in this study was able to grow in the presence of the ELQ-316 $\mathrm{IC}_{100}$ regardless of their initial PPE at $72 \mathrm{~h}$ $(P<0.05)$ (Fig. $4 \mathrm{a}-\mathrm{d})$. The same lack of parasite growth was observed after 8 days in the parasite rescue experiment, with the exception of B. caballi (Fig. $4 \mathrm{a}-\mathrm{d}$ ), independent of the starting PPE. A possible interpretation of these results is that the $B$. caballi strain used in this study may contain a mix of subpopulations of parasites, each one with distinct degrees of tolerance for ELQ-316. In contrast, the B. bovis, B. bigemina and T. equi strains used in these experiments appear to be composed of subpopulations that are highly susceptible to ELQ-316. It was beyond the scope of this study to investigate the mechanism involved in the susceptibility to the ELQ drugs. However, it may be speculated that such susceptibility 


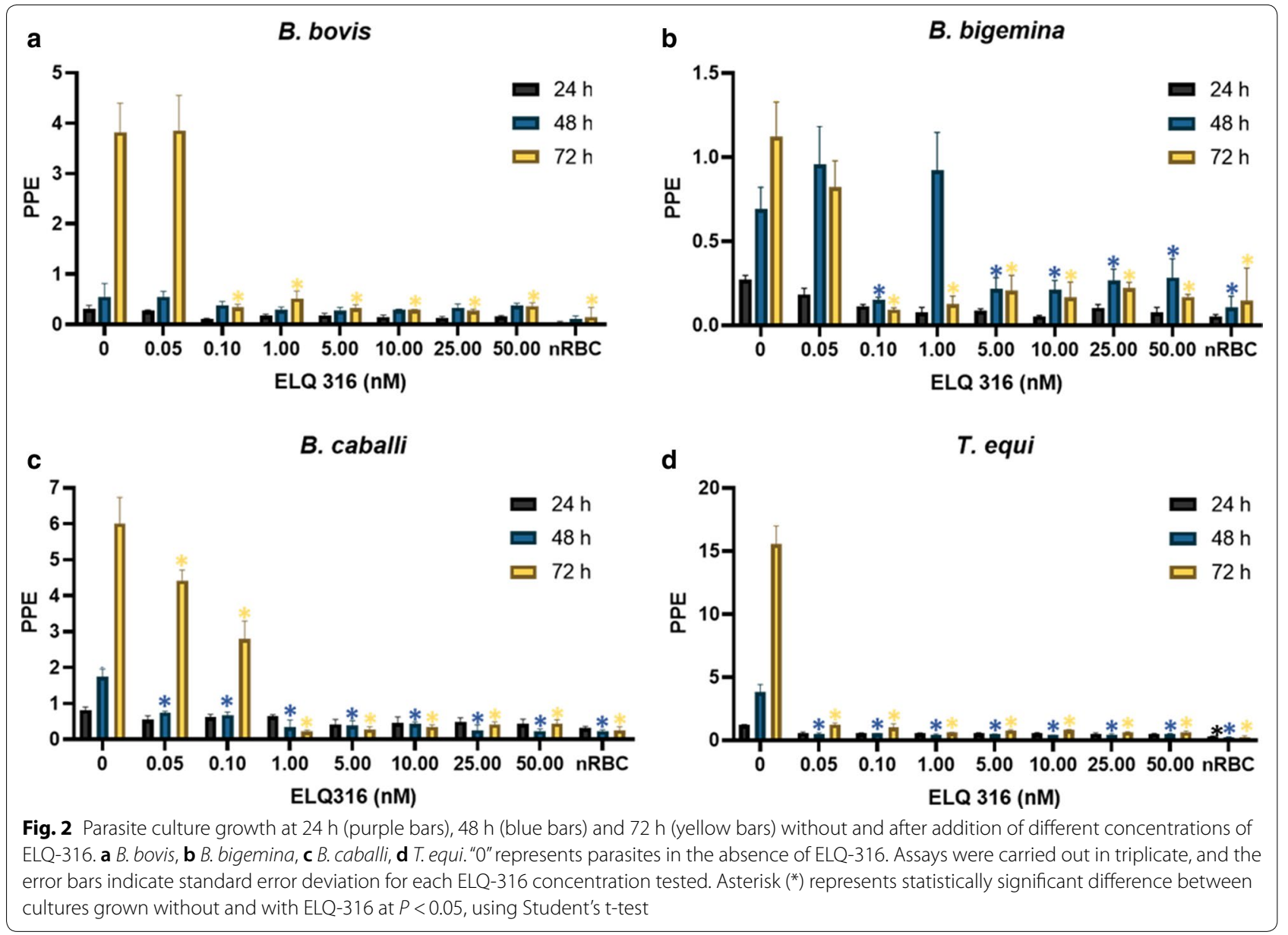

Table 1 Half maximal and maximal inhibitory concentrations of endochin-like quinolone (ELQ)-300 and ELQ-316 calculated for the four studied species of apicomplexan parasites at $72 \mathrm{~h}$ of culture

\begin{tabular}{|c|c|c|c|c|}
\hline \multirow[t]{2}{*}{ Species } & \multicolumn{2}{|l|}{ ELQ-300 } & \multicolumn{2}{|l|}{ ELQ-16 } \\
\hline & $\mathrm{IC}_{50}(\mathrm{nM})$ & $\mathrm{IC}_{100}(\mathrm{nM})$ & $\mathrm{IC}_{50}(\mathrm{nM})$ & $\mathrm{IC}_{100}(\mathrm{nM})$ \\
\hline Babesia bovis & $0.09 \pm 0.002$ & $4.2 \pm 0.10$ & 0.07 & $3.8 \pm 0.12$ \\
\hline B. bigemina & $0.37 \pm 0.19$ & $1.3 \pm 0.05$ & 0.05 & $3.0 \pm 0.15$ \\
\hline B. caballi & $0.19 \pm 0.04$ & $5.7 \pm 0.24$ & $0.1 \pm 0.006$ & $6.0 \pm 0.18$ \\
\hline Theilera equi & $0.04 \pm 0.003$ & $3.36 \pm 0.83$ & 0.002 & $1.0 \pm 1.55$ \\
\hline
\end{tabular}

Values in table are presented as the mean and standard deviation (SD) based on triplicates for each experiment.

$\mathrm{IC}_{50}$ and $\mathrm{IC}_{100}$, half maximal and maximal inhibitory concentrations, respectively

can be due to variations/mutations in the cytochrome $b c_{1}$ target sequence that affect ELQ binding, differential uptake or elimination of the drugs or a combination of these factors (Additional file 3: Figure S2) [29-31]. It was recently shown that genetic alterations in the $\mathrm{Q}_{i}$ binding site of the cytochrome $b c_{1}$ complex $(C y t b)$ of $B$. microti is associated with resistance to ELQ-316, which suggests that this cytochrome gene is as a potential target for the ELQ drugs [16]. Based on these observations, we performed alignment analysis of the Cytb genes of B. bovis, $B$. bigemina, B. caballi and T. equi together with the $B$. microti Cytb. Our results indicated full conservation of the two canonical $\mathrm{Q}_{\mathrm{o}}$ and $\mathrm{Q}_{\mathrm{i}}$ binding sites of Cytb in all sequences analyzed and a high level of amino acid identity, which ranged from 47.2 to $49.6 \%$ in comparison to B. microti (Fig. 2) (Additional file 2: Table S2). Overall, the results presented here set the rationale for further studies to alter and/or knockdown the Cytb gene in these parasites and evaluate its potential effect on the susceptibility or resistance to the ELQ drugs.

\section{ELQ-300 and ELQ-316 do not affect viability of equine and bovine PBMC}

Cytotoxic assays were performed to assess whether ELQ300 and ELQ-316 affect the viability of equine and bovine $\mathrm{PBMC}$, which we used as surrogates of nucleated vertebrate host cells. The cytotoxic assays were performed using the $\mathrm{IC}_{100}$ doses of ELQ-300 and ELQ-316 in in vitro 


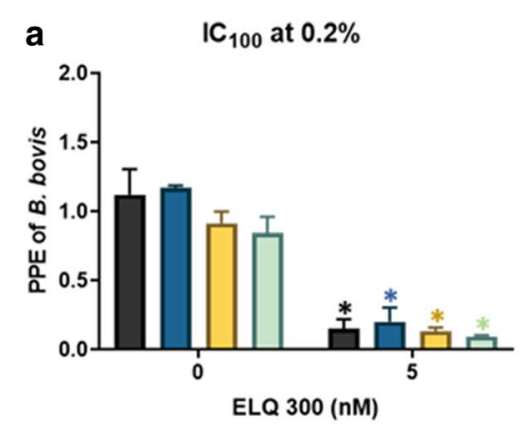

b $\quad \mathrm{IC}_{100}$ at $\mathbf{0 . 2 \%}$

口 $24 \mathrm{~h}$

口 $48 \mathrm{~h}$

口 $72 \mathrm{~h}$

口 8 days

$\mathrm{IC}_{100}$ at $\mathbf{2} \%$
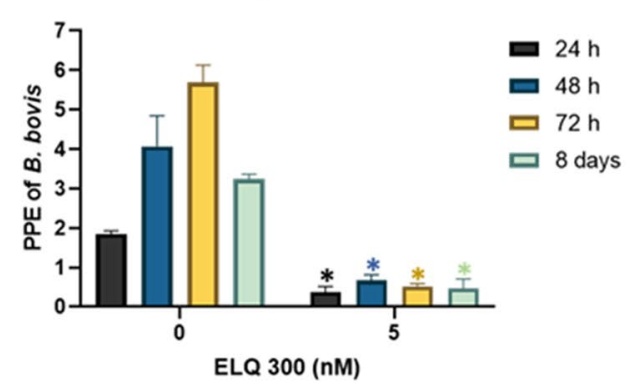

C

$\mathrm{IC}_{100}$ at $0.2 \%$

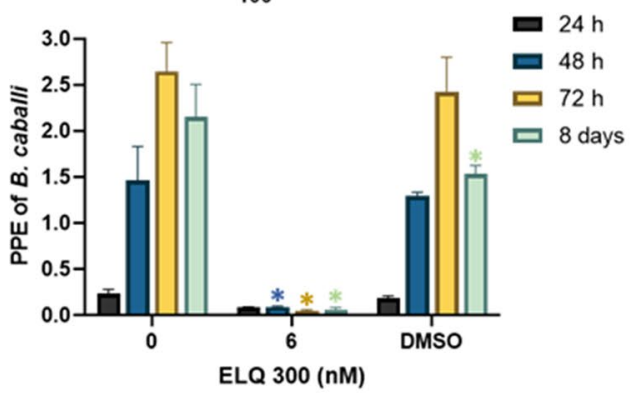

$I_{100}$ at $2 \%$

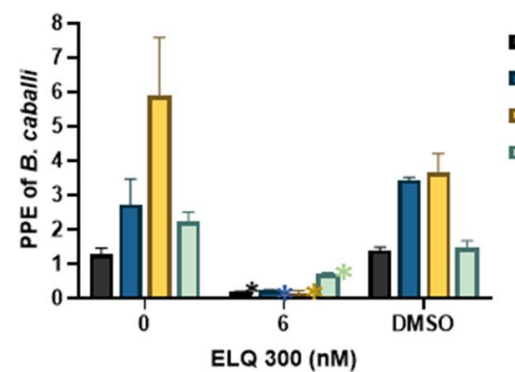

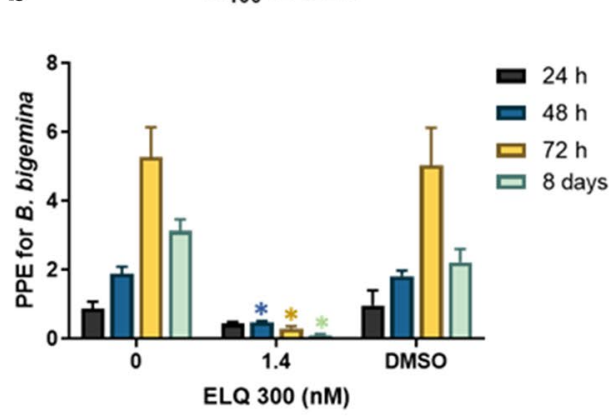

$\mathrm{IC}_{100}$ at $\mathbf{2 \%}$

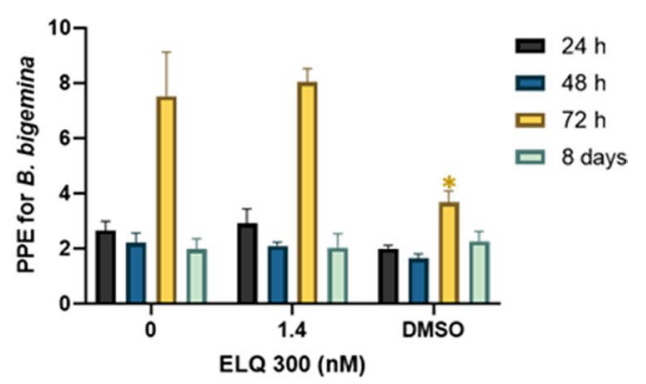

d

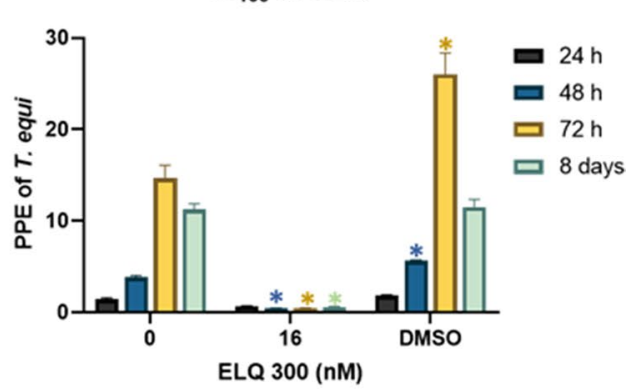

$\mathrm{IC}_{100}$ at $2 \%$

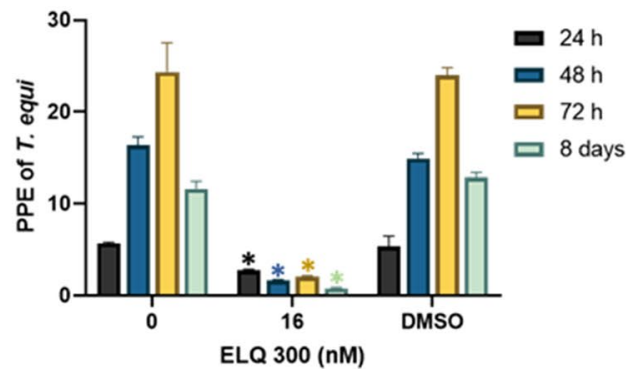

Fig. 3 Parasite culture growth at $24 \mathrm{~h}$ (purple bars), $48 \mathrm{~h}$ (blue bars), and $72 \mathrm{~h}$ (yellow bars) using $\mathrm{IC}_{100}$ of ELQ-300, and at 8 days (green bars). a $B$. bovis, b B. bigemina, c B. caballi, d T. equi. Upper panel of each figure part represents $0.2 \%$ PPE, lower panel 2\% PPE. "0" represents parasites grown without addition of ELQ-300. Assays were carried out in triplicate and the error bars indicate standard error deviation. Asterisk $\left(^{*}\right)$ represents statistically significant difference between cultures grown without and with ELQ-300 at $P<0.05$, using Student's t-test. $C_{50}$ and $/ C_{100}$ Half maximal and maximal inhibitory concentrations, respectively, DMSO dimethyl sulfoxide 

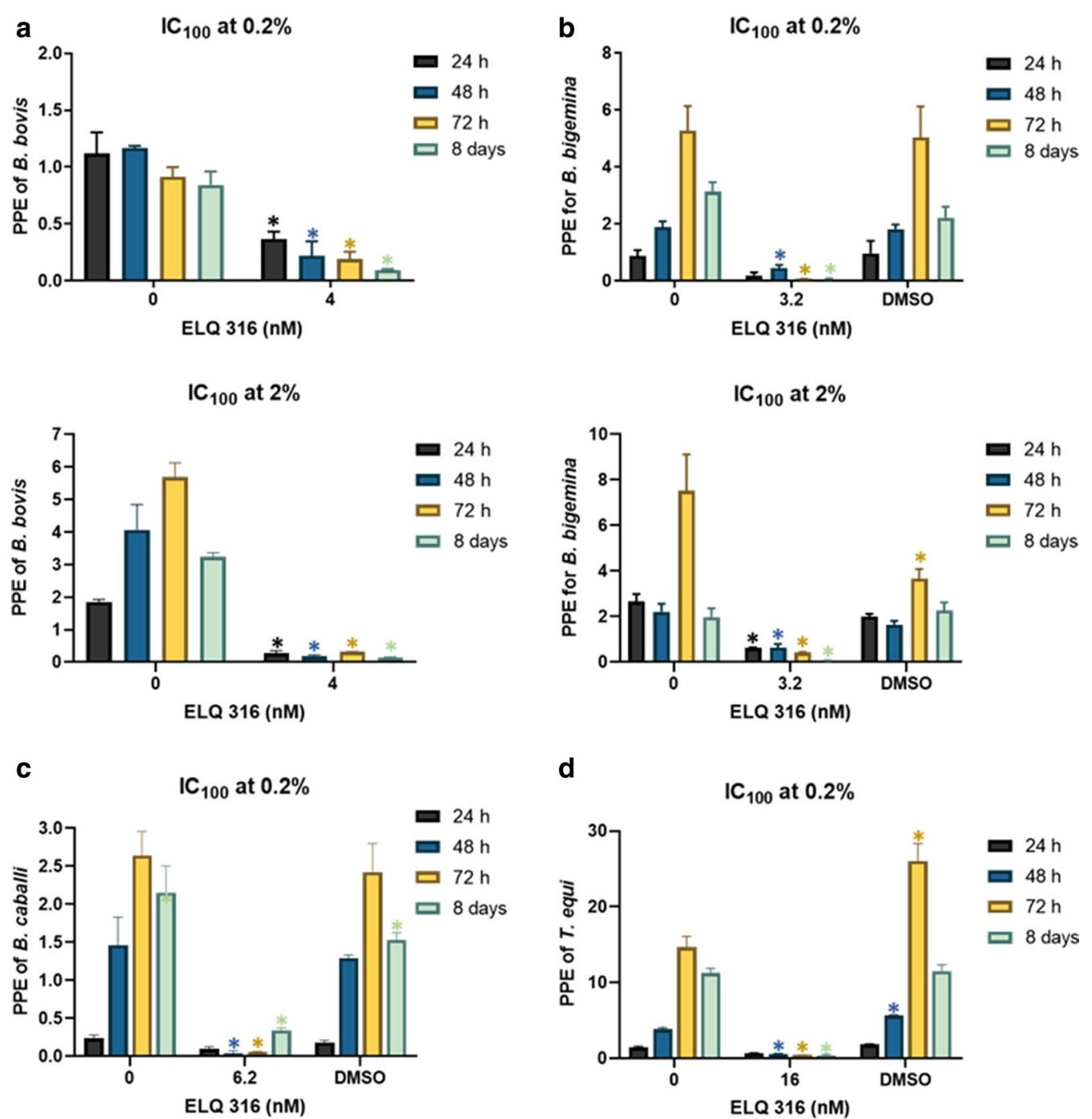

d

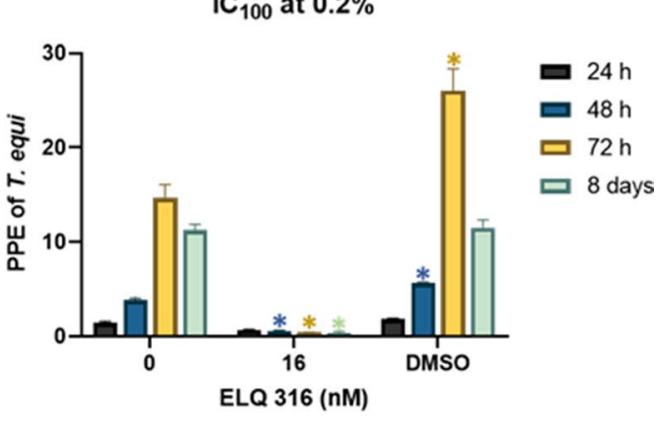

$\mathrm{IC}_{100}$ at $2 \%$

$I_{100}$ at $2 \%$
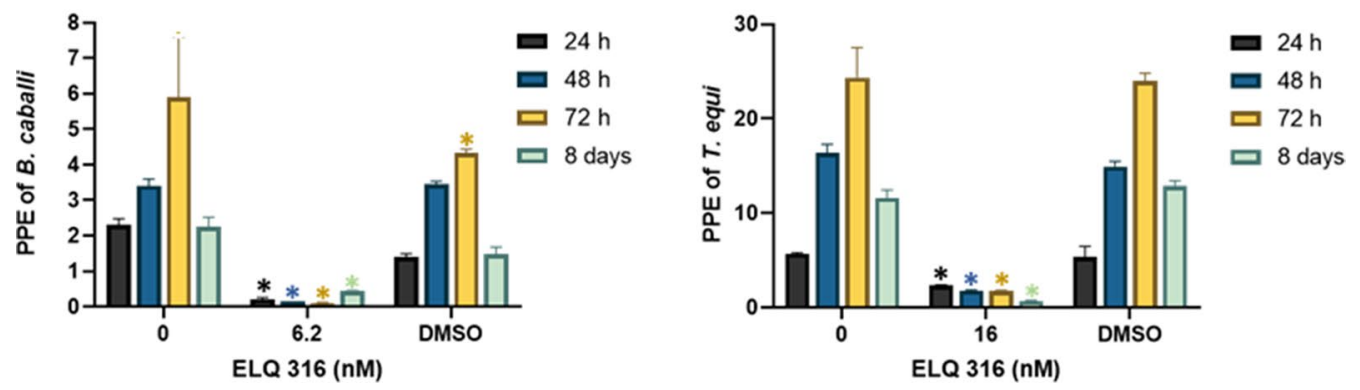

Fig. 4 Parasite culture growth at $24 \mathrm{~h}$ (purple bars), $48 \mathrm{~h}$ (blue bars), and $72 \mathrm{~h}$ (yellow bars) using the $\mathrm{IC}_{100}$ of ELQ-316, and at 8 days (green bars). a B. bovis, b B. bigemina, c B. caballi, d T. equi. Upper panel of each figure part represents $0.2 \%$ PPE and lower panel represents $2 \%$ PPE. " 0 " represents parasites grown without the addition of ELQ-316. Assays were carried out in triplicate and the error bars indicate standard error deviation. Asterisk ${ }^{*}$ ) represents statistically significant difference between cultures grown without and with ELQ-300 at $P<0.05$, using Student's t-test 
cultures. For the bovine PBMC experiment, the ELQ$300 \mathrm{IC}_{100}$ of $4.3 \mathrm{nM}$ and ELQ-316 $\mathrm{IC}_{100}$ of $3.92 \mathrm{nM}$ were used, respectively, whereas for the equine PBMC experiment, the ELQ-300 $\mathrm{IC}_{100}$ of $5.94 \mathrm{nM}$ and ELQ-316 $\mathrm{IC}_{100}$ of $6.18 \mathrm{nM}$ were used, respectively. Viability of PBMC was similar regardless of the presence or absence of parasite lethal doses of ELQ-300 or ELQ-316, strongly suggesting that cell viability was not compromised by any of these two drugs under the experimental conditions used in the assays (Fig. 5a, b). In addition, a significant increase $(P<0.05)$ in cell proliferation was observed in bovine and horse PBMC exposed to ConA for 24 and 48 h, respectively (Fig. 5a, b), indicating adequate sensitivity for the WST-1 proliferation assay used in this study. Taken together, results of the cell viability study revealed that ELQ-300 and ELQ-316, at their respective $\mathrm{IC}_{100}$, lack a significant toxic effect on bovine and horse PBMC cultured in vitro. Although the data presented here strongly suggest that these two drugs are appropriate candidates for the treatment of $\mathrm{BB}$ and $\mathrm{EP}$, it needs to be pointed out that we did not assess their effect in vivo and that our evidence on the effectivity and safety of ELQ-300 and ELQ316 was obtained from testing the effect of the drugs on

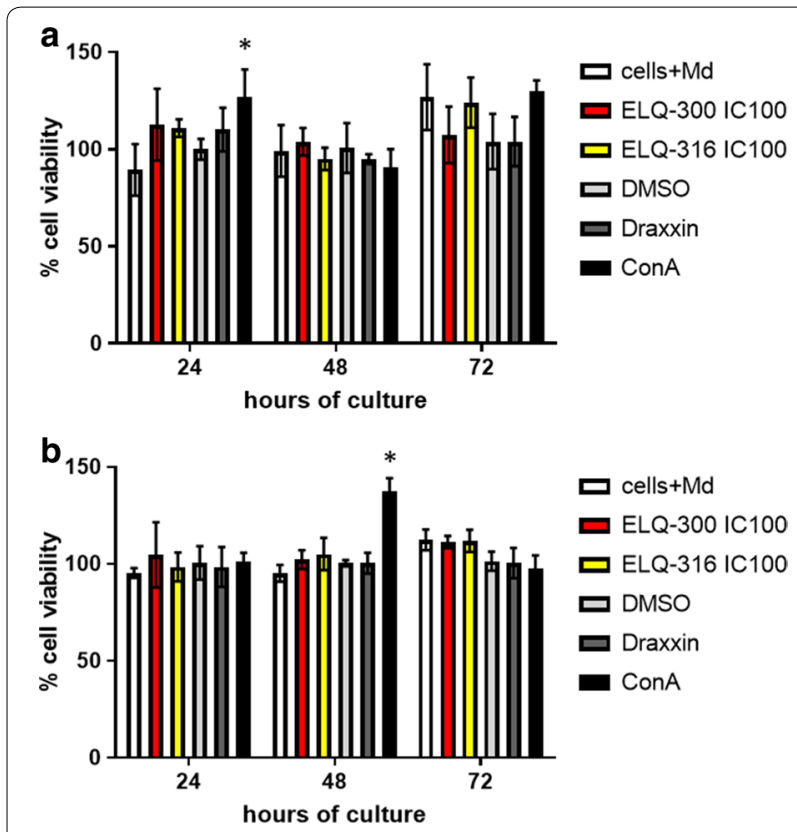

Fig. 5 Percentage of cell viability over a period of $72 \mathrm{~h}$ after incubation with the IC $\mathrm{C}_{50}$ and IC 100 of ELQ-300 and ELQ-316. Cells+Med Peripheral blood mononuclear cells (PBMC) cultivated without the addition of the ELQ compounds. Cells in cultured in DMSO and Draxxin ${ }^{\circledR}$ were used as a negative control; concanavalin A (ConA) was used as a positive control for cell proliferation. a Bovine PBMC, b horse PBMC. Bovine and horse PBMC assays were carried out in triplicate and the error bars indicate standard error deviation. Asterisk $\left(^{*}\right)$ represents statistically significant differences compared to PBMC cultivated in medium only at $P<0.05$, using Student's t-test parasites growing in culture. In addition, investigation of the mechanism of action of ELQ-300 and ELQ-316 in the parasites studied herein was also beyond the scope of this study, and this aspect needs further examination.

\section{Conclusions}

Overall, the results presented here demonstrate that both of the drugs tested in this study, ELQ-300 and ELQ-316, are efficient in inhibiting the growth of in vitro-cultured B. bovis, B. bigemina, B. caballi and T. equi. Importantly, the $\mathrm{IC}_{100}$ doses of the ELQ drugs did not significantly affect the viability of in vitro-cultured cattle and horse PBMC. Collectively, the findings of this study strongly suggest that ELQ-300 and ELQ-316 can be potentially effective and safe candidates for the development of novel therapies to control BB and EP. However, it will be important to confirm their mechanisms of action, as well as the drugs's potential to select for resistant strains. Further studies in vivo in horses and bovines are needed to evaluate the efficacy of ELQ-300 and ELQ-316 against acute and chronic BB and EP.

\section{Supplementary information}

Supplementary information accompanies this paper at https://doi. org/10.1186/s13071-020-04487-3.

Additional file 1: Fig. S1. Chemical structures of ELQ-300 (a) and ELQ-316 (b).

Additional file 2: Table S1. $I C_{50}$ calculated for different compounds tested against in vitro in B. bovis, B. bigemina, B. caballi and T. equi cultures. Table S2. Amino acid percentage identity of cytochrome bc1 complex (Cytb) of Babesia bovis, B. bigemina, B. caballi, and Theileria equi in comparison to B. microti.

Additional file 3: Fig. S2. Alignment of amino acid sequences of the cytochrome bc1 complex (Cytb) of B. bovis (GenBank accession YP_001504108), B. bigemina (GenBank accession BAl66164.1), B. caballi (GenBank accession BAl66167.1), T. equi (GenBank accession XP_025033545.1), and B. microti (GenBank accession MT114078).

\section{Abbreviations}

Con A: Concanavalin A; DMSO: Dimethyl sulfoxide; ELQ: Endochin-like quinolones; HE: Hydroethidine; IC: Inhibitory concentration; PBMC: Peripheral blood mononuclear cells; PBS: Phosphate buffer saline; PPE: Percentage of parasitized erythrocytes.

\section{Acknowledgements}

The authors would like to acknowledge Paul Lacy for his help with the in vitro B. bovis, B. bigemina and B. caballi cultures and culture supplies; Johanatan Hyunki and Ji Heon Lee for checking PPE on smears; Jacob Laughery for providing uninfected bovine erythrocytes and serum; Lowell Kappemeyer for providing the initial in vitro B. caballi and T. equi cultures and the uninfected equine erythrocytes; and Megan Blauert for animal handling and blood draw.

\section{Author's contribution}

MGS, RGB and CES conceived and designed the study. MGS and RGB performed the inhibitory assays and microscopy experiments, analyzed the data and drafted the manuscript. MGS and RGB performed the flow cytometry assay and cytotoxic assay. JSD, MKR, SP, RW and AN manufactured and provided endochin-like quinolones and provided technical guidance regarding 
their use. MGS, RGB, CES, JSD and MKR wrote the manuscript. All authors read and approved the final manuscript.

\section{Funding}

This work was funded by the US Department of Agriculture-Agriculture Research Service Current Research Information System Project No. 209032000-039-00D, and the International Development Research Centre (IDRC) (Canada) project 108525-001. MKR's laboratory receives financial support from the US Department of Veterans Affairs, Veterans Health Administration, Office of Research and Development Program Award (number i01 BX003312). MKR is a recipient of a VA Research Career Scientist Award (14S-RCS001). Research reported in this publication was also supported by the US National Institutes of Health under award number Al100569 (MKR) and by the US Department of Defense Peer Reviewed Medical Research Program (Log \# PR181134 (MKR), and the National Institute of Allergy and Infectious Diseases under award number R01Al141412. This work was also funded by Career Development Award BX002440 and VA Merit Review Award BX004522 to JSD from the US Department of Veterans Affairs Biomedical Laboratory Research and Development.

\section{Availability of data and materials}

The datasets supporting the conclusions of this article are included within the article and its Additional files 1,2 and 3 .

\section{Ethics approval and consent to participate}

Not applicable.

\section{Consent for publication}

Not applicable.

\section{Competing interests}

The authors declare that they have no competing interests.

\section{Author details}

${ }^{1}$ Department of Veterinary Microbiology and Pathology, College of Veterinary Medicine, Washington State University, Pullman, WA, USA. ${ }^{2}$ Oregon Health and Science University, 3181 SW Sam Jackson Blvd., Portland, Oregon 97239, USA. ${ }^{3}$ VA Portland Health Care System, 3710 SW US Veterans Hospital Road, Portland, OR 97239, USA. ${ }^{4}$ Animal Disease Research Unit, Agricultural Research Service, USDA, WSU, Pullman, WA, USA.

Received: 11 August 2020 Accepted: 17 November 2020 Published online: 03 December 2020

\section{References}

1. Uilenberg G. International collaborative research: Significance of tickborne hemoparasitic diseases to world animal health. Vet Parasitol. 1995;57:19-41.

2. Wise LN, Kappmeyer LS, Silva MG, White SN, Grause JF, Knowles DP. Verification of post-chemotherapeutic clearance of Theileria equi through concordance of nested PCR and immunoblot. Ticks Tick Borne Dis. 2018;9:135-40.

3. Wise LN, Pelzel-McCluskey AM, Mealey RH, Knowles DP. Equine piroplasmosis. Vet Clin N Am-Equine. 2014;30:677-93.

4. Florin-Christensen $M$, Suarez CE, Rodriguez AE, Flores DA, Schnittger L. Vaccines against bovine babesiosis: where we are now and possible roads ahead. Parasitol. 2014;1-30. https://doi.org/10.1017/S003118201 4000961.

5. Schnittger L, Rodriguez AE, Florin-Christensen M, Morrison DA. Babesia: A world emerging. Infect Genetics Evol. 2012;12:1788-809.

6. Onyiche TE, Suganuma K, Igarashi I, Yokoyama N, Xuan X, Thekisoe O. A review on equine piroplasmosis: epidemiology, vector ecology, risk factors, host immunity, diagnosis and control. Int J Environ Res Public Health. 2019;16:1736.

7. Kappmeyer LS, Thiagarajan M, Herndon DR, Ramsay JD, Caler E, Djikeng A, Gillespie JJ, et al. Comparative genomic analysis and phylogenetic position of Theileria equi. BMC Genomics. 2012;13:603.
8. Siddra A, Hines JB, Mealey RH, Call DR, Graça T. Exposure to ambient air causes degradation and decreased in vitro potency of buparvaquone and parvaquone. Vet Parasitol X. 2020;3:1000023.

9. Suarez CE, Noh S. Emerging perspectives in the research of bovine babesiosis and anaplasmosis. Vet Parasitol. 2011;180:109-25.

10. Kuttler KL, Johnson LW. Chemoprophylactic activity of imidocarb, diminazene and oxytetracycline against Babesia bovis and B. bigemina. Vet Parasitol. 1986;2:107-18.

11. Yamasaki M, Watanabe N, Idaka N, Yamamori T, Otsuguro KI, Uchida N et al. Intracellular diminazene aceturate content and adenosine incorporation in diminazene aceturate-resistant Babesia gibsoni isolate in vitro. Exp Parasitol. 2017;183:92-8.

12. Schwint ON, Ueti MW, Palmer GH, Kappmeyer LS, Hines MT, Cordes RT, et al. Imidocarb dipropionate clears persistent Babesia caballi infection with elimination of transmission potential. Antimicrob Agents Chemother. 2009;53:4327-32.

13. Yeruham I, Pipano E, Davidson M. A field strain of Babesia bovis apparently resistant to amicarbalide isethionate. Trop Anim Health Prod. 1985;17:29-30

14. Miley GP, Pou S, Winter R, Nilsen A, Li Y, Kelly JX, et al. ELQ-300 prodrugs for enhanced delivery and single-dose cure of malaria. Antimicrob Agents Chemother. 2015;59:5555-60.

15. van Schalkwyk DA, Riscoe MK, Pou S, Winter RW, Nilsen A, Duffey M, et al. Novel endochin-like quinolones exhibit potent in vitro activity against Plasmodium knowlesi but do not synergize with proguanil. Antimicrob Agents Chemother. 2020;64:e02549-e2610.

16. Winter R, Kelly JX, Smilkstein MJ, Hinrichs D, Koop DR, Riscoe MK. Optimization of endochin-like quinolones for antimalarial activity. Exp Parasitol. 2011;127:545-51.

17. Winter RW, Kelly JX, Smilkstein MJ, Dodean R, Hinrichs D, Riscoe MK. Antimalarial quinolones: synthesis, potency, and mechanistic studies. Exp Parasitol. 2008;118:487-97.

18. Frueh L, Li Y, Mather MW, Li Q, Pou S, Nilsen A, et al. Alkoxycarbonate ester prodrugs of preclinical drug candidate ELQ-300 for prophylaxis and treatment of malaria. ACS Infect Dis. 2017;3:728-35.

19. Nilsen A, LaCrue AN, White KL, Forquer IP, Cross RM, Marfurt J, et al. Quinolone-3-diarylethers: a new class of antimalarial drug. Sci Transl Med. 2013;5:177ra137.

20. Lawres LA, Garg A, Kumar V, Bruzual I, Forquer IP, Renard I, et al. Radical cure of experimental babesiosis in immunodeficient mice using a combination of an endochin-like quinolone and atovaquone. J Exp Med. 2016;213:1307-18.

21. Doggett JS, Nilsen A, Forquer I, Wegmann KW, Jones-Brando L, Yolken $\mathrm{RH}$, et al. Endochin-like quinolones are highly efficacious against acute and latent experimental toxoplasmosis. Proc Natl Acad Sci USA. 2012;109:15936-41.

22. Avarzed A, Igarashi I, Kanemaru T, Hirumi K, Omata Y, Saito A, et al. Improved in vitro cultivation of Babesia caballi. J Vet Med Sci. 1997;59:479-81.

23. Levy MG, Ristic M. Babesia bovis: continuous cultivation in a microaerophilous stationary phase culture. Science. 1980;207:1218-20.

24. Vega CA, Buening GM, Green TJ, Carson CA. In vitro cultivation of Babesia bigemina. Am J Vet Res. 1985:46:416-20.

25. Zweygarth E, Just MC, de Waal DT. Continuous in vitro cultivation of erythrocytic stages of Babesia equi. Parasitol Res. 1995;81:355-8.

26. Silva MG, Villarino NF, Knowles DP, Suarez CE. Assessment of Draxxin((R)) (tulathromycin) as an inhibitor of in vitro growth of Babesia bovis, Babesia bigemina and Theileria equi. Int J Parasitol Drugs Drug Resist. 2018:8:265-70.

27. Wyatt CR, Goff W, Davis WC. A flow cytometric method for assessing viability of intraerythrocytic hemoparasites. J Immunol Methods. 1991;140:23-30.

28. Eberhard N, Balmer V, Muller J, Muller N, Winter R, Pou S, et al. Activities of endochin-Like quinolones against in vitro cultured Besnoitia besnoiti tachyzoites. Front Vet Sci. 2020;7:96. https://doi.org/10.3389/fvets .2020.00096.

29. Alday PH, Bruzual I, Nilsen A, Pou S, Winter R, Ben Mamoun C, et al. Genetic evidence for cytochrome $b$ Qi site inhibition by 
4(1H)-quinolone-3-diarylethers and antimycin in Toxoplasma gondii. Antimicrob Agents Chemother. 2017;61:e01866-e1916.

30. McConnell EV, Bruzual I, Pou S, Winter R, Dodean RA, Smilkstein MJ, et al. Targeted structure-activity analysis of endochin-like quinolones reveals potent Qi and Qo site inhibitors of Toxoplasma gondii and Plasmodium falciparum cytochrome bc1 and identifies ELQ-400 as a remarkably effective compound against acute experimental toxoplasmosis. ACS Infect Dis. 2018:4:1574-84.
31. Silva MG, Domingos A, Esteves MA, Cruz ME, Suarez CE. Evaluation of the growth-inhibitory effect of trifluralin analogues on in vitro cultured Babesia bovis parasites. Int J Parasitol Drugs Drug Resist. 2013;3:59-68.

\section{Publisher's Note}

Springer Nature remains neutral with regard to jurisdictional claims in published maps and institutional affiliations.
Ready to submit your research? Choose BMC and benefit from:

- fast, convenient online submission

- thorough peer review by experienced researchers in your field

- rapid publication on acceptance

- support for research data, including large and complex data types

- gold Open Access which fosters wider collaboration and increased citations

- maximum visibility for your research: over $100 \mathrm{M}$ website views per year

At BMC, research is always in progress.

Learn more biomedcentral.com/submissions 